\title{
Potential of Modelica for the creation of digital twins
}

\author{
Stefan Vöth \\ Prof. Dr.-Ing, Technische Hochschule Georg Agricola (THGA), Germany \\ Maria Vasilyeva \\ PhD, Associate professor, St. Petersburg Mining University, Russia
}

\begin{abstract}
Digitalization is on path of fast development in all spheres of society. As an outcome the requirements on modeling of systems are increasing. This applies with regard to quantity and quality. In this context the tools for modeling have to be selected. Considering modeling a possible choice is the language Modelica. This language comprises different advantages, finally supporting safe use and effective reuse of already developed components. Especially Modelica is an efficient approach to model acausal interaction between objects, which practically occurs fairly in every system, in technical systems typical for mechanical engineering e.g. The text comes with possible views on the term digital twin, which seems not to be defined exactly. It continues with Modelica, its properties and advantages for the creation of digital twins. Summarized the text should give some support to get acquainted, to evaluate and to decide on the usage of Modelica.
\end{abstract}

\section{DIGITAL TWIN}

The headline mentions two keywords, which have to be understood: Modelica and digital twin. A digital twin is a representation of some object or system of objects in the digital sphere. Certainly the question can be stated, whether this description is sufficient, but it at least depicts a minimum requirement. The object may be material or not material. It may represent products, production systems or services. Such representation will not represent „all“ properties of that object, but some selection of properties. Thus an infinite bunch of representations of a digital twin may exist. The choice of the suitable representation is a main task working on digital twins.

A digital twin may be used for different tasks along the lifecycle of a product, from planning to recycling. The digital twin may be built up independant of the question, whether the object already exists or is planned so far only. History first came up with digital twins for not existing objects to handle early phases of the lifecycle. Today motivation is increasing to support later phases oft he lifecycle by digital twins as well.

Possible areas of application are: The product may be developed just in virtual sphere. Production processes may be tested and optimized there as well. The product may be tested without production of a real prototype. Personnel may be trained on the job without an equipment already existing. Modifications of products and processes may be prepared in digital sphere.

Especially tests in digital sphere comprise severeal advantages: Theoretical and potentially dangerous situations may be analyzed. Tests potentially time, money or space consuming or even not possible to be executed get into focus of availability. In virtual world probably a broader bunch of tests will be executed than in real world. These tests during different project phases with models of different detailing of description can lead to a deepend learning and understanding on theoretical basis.

Preparations in virtual sphere deliver the potential of flawless processes including time and money savings. A focus may be the costly commissioning, e.g. in plant construction. But also the risk of wrong forecasts due to insufficient systems descriptions. 
The digital twin consists of properties and behaviour. Properties are represented by data, behaviour is represented by algorithms and/or equations. Algorithms and equations are associated with causal and acausal modeling.

The digital twin may be coupled with outside world. Via input channels and output channels data may be gained or may be supplied by the digital twin. A special channel are internet connections, which allow distant data exchange. This is one property enabling systems for condition dependant flexible control, called among others „Industry 4.0“.

Along lifetime of the product the idea of a digital twin may be to get a continous digital thread. On basis of operational data the digital twin gathers data. The data may be analyzed, e.g. to execute optimization in order improve usage of the product. In advantage to planning phase this gives the chance of improved optimization on basis of real operational data. Some higher degree of optimization will lead to the modification of the descriptions of properties and beahviour of the system itself. In this case the digital twin is rather dynamically devloping than a static despription. The developed digital twin will give a dramatically enhanced basis for future planning.

Furthermore the data may be analyzed with regard to future behaviour of the system. This gives the chance of enhancement of predictive maintenacne approaches.

This proposes a holistic view of the whole lifecycle. A complete data exchange between all the cycle stages would support variety of new options. This idea introduces several new nontechnical questions on supplier-customer-relation, intercompany data exchange, national and international legislation and data security.

\section{MODELICA}

The headline mentions two keywords, which have to be understood: Modelica and digital twin. Modelica is a programming language especially suitable for the modeling of mathematical models. It can be used for (nearly) any kind of mathematical model. A lot of applications consider technical aspects, but Modelica is not restricted to such. The modeling of social systems or financial systems are options e.g. In fact Modelica has unrestricted multidomain modeling capability.

Different domains may be coupled easily. E.g. using the control domain, the electric domain and the mechanical domain an electro-mechanical drivetrain may be modeled. Objects belonging to a domain are typically collected in toolboxes. Various toolboxes are available, platform built-in, external of platforms, free and commercial.

A core property of Modelica ist he usage of equations. Equations indicate acausal modeling including a flexible data flow direction (Figure 1). This is of importance, as many systems comprise bidirectional flow of information, which can be easily modeled by equations. Assignments, which model unidirectional flow of information (causal modeling), are also supported by Modelica, e.g. in algorithmic sections. Causal and acausal approaches may be used next to each other in one model. This enables a stable object representation.

Modelica is an object-oriented language. This supports the structuring of systems and objects and the reuse of objects. This aspect empowers the toolboxes available.

The focus of Modelica is on modeling. Platforms support the automatic transfer of the model to a mathematical model and finally to an executable program. Basically there is no dealing with differential-algebraic equations, conversion of equations, numerical solvers, code generation and post-processing. The idea is to give the chance to focus on the engineering task rather than programming tasks, in order to increase the productivity of the modeling process.

A further property of Modelica is to associate units with variables. This enables the automatic test on unit integrity of the model.

As Modelica is object-oriented it is recommended, to decompose a system in components and to describe them as software objects. The decomposition should be executed from high complexity on top level to low complexity on the suitable low level. The number of levels depend on the system character. This approach is especially recommended using existing model classses. If no (fitting) model classes are available at all, a bottom up approach may be 


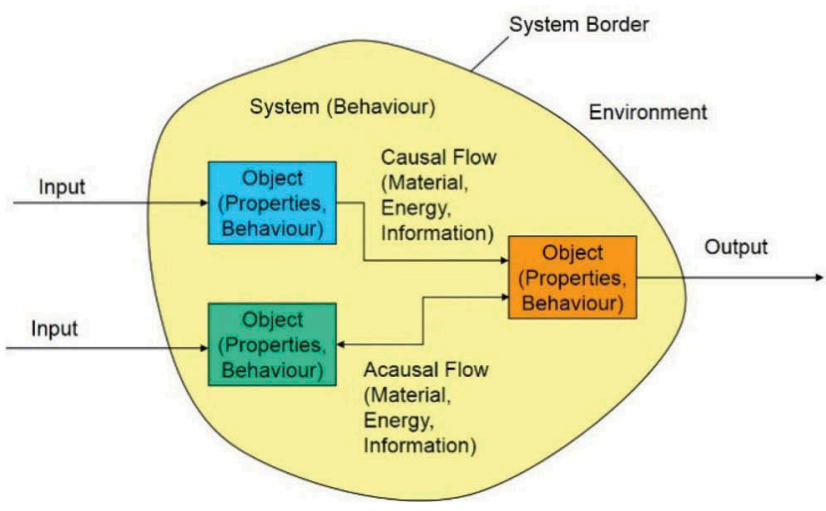

Figure 1. Properties of a system in general.

more suitable. Maybe existing model classes can be applied, maybe new model classes have to be developed. The connection of objects between each other is realized by connector classes. The connector classs defines the data interchanged via the interface between the objects. Connector classes are members of object classes. Connectors with compatible data interface can be connected to each other (Figure 2).

Open-source tools and commercial tool for working with Modelica are available both.

\section{APPLICATIONS}

At THGA Modelica is used to build up digital twins.

First application is the modeling of the behaviour of hoisting systems of fast running cranes. The models are used to plan the parametrization of hoists in order to optimize the loading of drivetrain components.

Second application is the modeling of ventilation systems of mines. Target of this application is the energy efficient control of air mass flows.

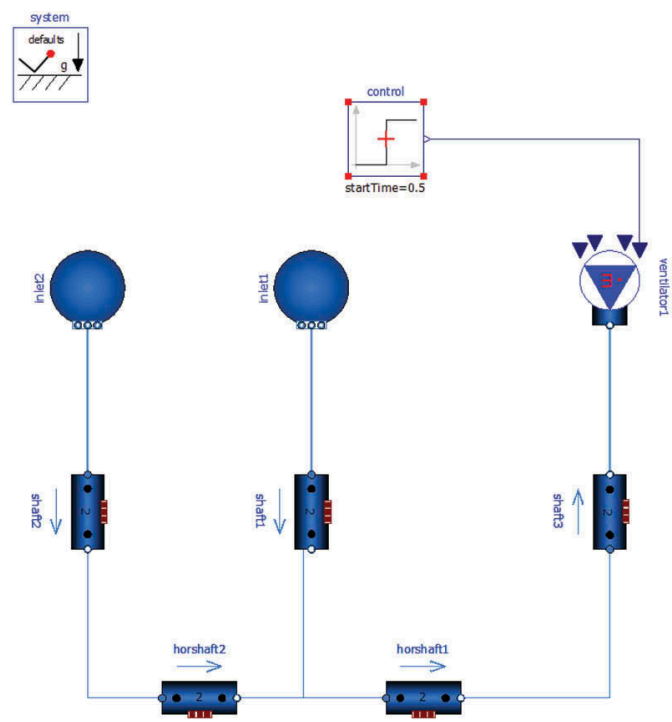

Figure 2. Objects, interfaces and connections as elements of a model for the ventialtion of an underground mine. 


\section{CONCLUSIONS}

There are different languages for modeling objects in digital sphere. One option is Modelica, which comprises several advantages:

- Multidomain modeling. No restrictions to the definition of new domains.

- Model structuring and model reuse supported by object-orientation.

- Supporting of causal modeling and acausal modeling as well.

- Support of units for the description of parameters and variables.

- Focussing on modeling instead of coding.

- Textual and visual tools for programming available on platforms.

- Diverse resources available, free and commercial.

\section{REFERENCES}

Chrisofakis E, Junghanns A: Faster development of automotive control software with Modelica and FMI, 2013.

Elmqvist H: Modelica Evolution - from my Perspective, Proceedings of the 10th International ModelicaConference, Lund, 2014.

Elshekih: Modelica_based Computational Tools for Sensitivity Analysis via Automatic Differentiation, Dissertation, RWTH Aachen, 2012.

Fritzson P: Introduction to Modeling and Simulation of Technical and Physical Systems with Modelica. IEEE Press 2011.

Fritzson P, Rogovchenko O: Introduction to Object-Oriented Modeling, Simulation and Control with Modelica, Tutorial, MODPROD, 2012.

Fritzson P: Principles of Object-Oriented Modeling and Simulation with Modelica 3.3: A Cyber-Physical Approach, John Wiley \& Sons Inc, 2nd edition, 2015.

Junglas P: System-Dynamics-Diagramme als "Physical Modeling" - eine Frage der Kausalität, 2014.

Maurer W: Modelica simuliert komplexe Systeme, Swiss Engineering, 2011.

Maurer W: Modeling of elastic buffers with DyMoRail, 2014.

Norrefeld V, Obelz S, Wirth F: Aufbau einer Modelica Modellbibliothek, BauSIM, 2014.

Ochel L: Petri-Netz-basierte Simulation biologischer Prozesse mit OpenModelica, Bielefeld, 2016.

Peter Beater: Modellbildung und Simulation technischer Systeme mit Modelica 3: Eine kurze Einführung für Ingenieure und Studenten, Books on Demand, 1. Auflage, 2016.

Tatar M, Schaich R: Automated test of the AMG speedshift DCT control software, Berlin, 2010.

Thiele B.A: Framework for Modelica based Function Development, Dissertation, TU München, 2015.

Waurich V, Will F: Simulation der automatisierten Schaufelbefüllung eines Radladers mit Modelica, 2018.

Weck O, Chodas M, Shougarian N: Fundamentals of systems Engineerinmg, MIT, 2015. 